\title{
Trabecular meshwork bypass tube shunt: initial case series
}

\section{Spiegel, K Kobuch}

Br J Ophthalmol 2002;86:1228-1231

Aims: This study describes a prospective consecutive case series of the initial six eyes of five patients undergoing implantation of the trabecular bypass tube shunt.

Methods: A prospective consecutive case series. The initial six eyes of five patients with uncontrolled open angle glaucoma who had never previously undergone ocular surgery. Implantation of the trabecular bypass tube shunt measuring $150 \mu \mathrm{m}$ outer diameter and $50 \mu \mathrm{m}$ inner diameter was performed with the distal end placed in Schlemm's canal and the proximal end in the anterior chamber. The main outcome measures were visual acuity, intraocular pressure, glaucoma medication use.

Results: The tube was successfully implanted in five of six eyes. In four eyes longer term follow up of 5-9 months showed no loss of visual acuity with decreased intraocular pressure from preoperative levels (mean 23.4-16.5 mm $\mathrm{Hg}$ ) and reduced requirement of glaucoma medications (mean 3-0.5). In a subset of two eyes, there was no measured increase in aqueous flare or reduction of endothelial cell count. In one eye the tube was explanted because of presumed misplacement by excessive bleeding during surgery. Two eyes showed a diffuse bleb.

Conclusions: This study reports the initial experience with a novel approach to surgical glaucoma therapy. This very small tube allows a direct communication to be established between the anterior chamber and Schlemm's canal, effecting a trabecular bypass. In this small number of eyes this procedure reduced intraocular pressure and the need for glaucoma medications without appreciable side effects.

G uarded filtration surgery is currently the standard procedure performed in open angle glaucoma patients requiring incisional surgery. Cairns ${ }^{1}$ first described trabeculectomy as a method for aqueous humour to bypass the trabecular meshwork and directly enter the cut ends of Schlemm's canal. However, experience has revealed that most commonly, success of this procedure is dependent on the presence of a filtering bleb. ${ }^{2}$ Non-penetrating surgery has recently emerged as a potential alternative to traditional guarded filtration surgery in the surgical treatment of glaucoma. ${ }^{3}{ }^{4} \mathrm{~A}$ bypass of the trabecular meshwork allowing an alternative path for aqueous to access Schlemm's canal from the anterior chamber is the underlying basis for nonpenetrating glaucoma surgery including deep sclerectomy and viscocanalostomy. The former procedure removes the outer wall of Schlemm's canal and excises deep sclera and peripheral cornea while the latter also unroofs the canal but additionally dilates the canal with viscoelastic material. These techniques have demonstrated the potential to control intraocular pressure (IOP) with a reduction in the risks present with the bleb associated with traditional filtration surgery. ${ }^{4-6}$ Unfortunately, the usefulness of these procedures is

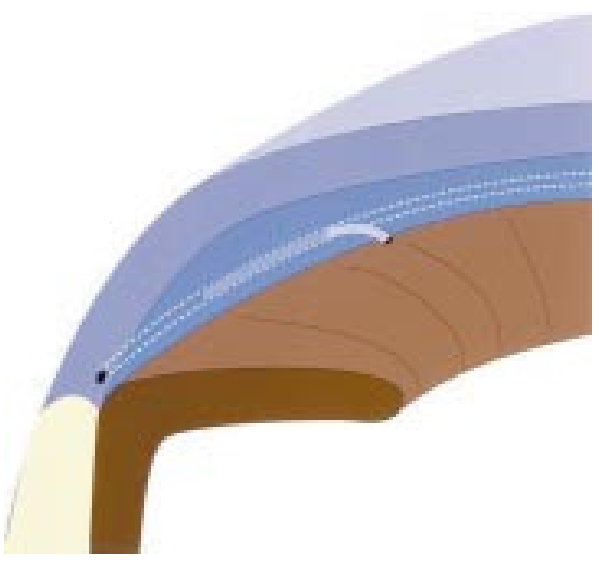

Figure 1 Schematic drawing of the bypass tube shunt in situ.

often limited by their unpredictable success in lowering IOP and technical difficulty. ${ }^{7-9}$

The reason for this interest in trabecular bypass is based on the evidence that the elevated intraocular pressure in glaucoma is due to an increased resistance to outflow and that the majority of resistance is present in the juxtacanalicular connective tissue of the trabecular meshwork including the inner wall of Schlemm's canal. ${ }^{11}{ }^{11}$ However, in one study, 35\% of resistance was found to be distal to the inner wall of Schlemm's canal. ${ }^{12}$ Even at its theoretical best, pressure reduction exclusively by trabecular bypass would only be able to achieve an intraocular pressure approximating episcleral venous pressure.

Previously we reported a new alternative approach to trabecular bypass, describing the technique of a Schlemm's canal implant trabecular bypass tube allowing direct communication between the anterior chamber and Schlemm's $\operatorname{canal}^{13}$ (Fig 1). This potentially addresses many of the limitations with non-penetrating glaucoma surgery, including the technical difficulty of performing the procedure, the potential direct microperforation of the trabecular meshwork, and the permeability (or lack thereof) of Descemet's membrane to aqueous. ${ }^{14}$ It provides another technique to directly address the presumed site of pathology in open angle glaucoma-the resistance of the trabecular meshwork and the inner wall of Schlemm's canal with the potential advantage of minimising the formation of filtration blebs and their potential complications.

\section{Design}

This study is an interventional case series describing an initial consecutive case series of the initial six eyes of five patients undergoing implantation of the trabecular bypass tube shunt.

\section{MATERIALS AND METHODS}

This was a prospective consecutive case series. 


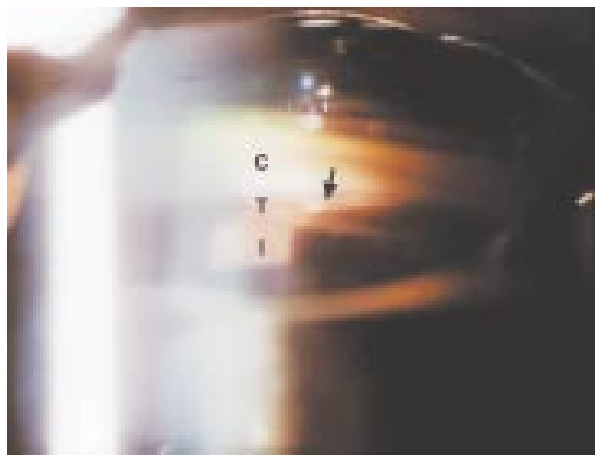

Figure 2 Gonioscopic view of the tube (arrow) emerging just anterior to the trabecular meshwork. $\mathrm{C}=$ cornea, $\mathrm{T}=$ trabecular meshwork, I = iris (9 months postoperative).

\section{Patients}

Inclusion criteria included open angle glaucoma as determined by the presence of typical optic nerve and visual field damage, uncontrolled IOP despite maximum tolerated medical therapy, and no history of previous ocular surgery including argon laser trabeculoplasty. Exclusion criteria included vision worse than $20 / 40$ in the fellow eye or corneal abnormalities interfering with reliable applanation tonometry.

The tenets of the World Medical Association Declaration of Helsinki were followed; institutional review board approval was obtained; informed consent was obtained from all subjects after the experimental nature of the procedure had been fully explained.

\section{Observation procedures}

A complete ophthalmological examination was performed before surgery. Preoperative assessment included visual acuity, applanation IOP measurement, and in selected eyes, aqueous flare measurement (Kowa FM 500 Laser Flare Meter, Kowa Optimed, Inc, Torrance CA, USA), specular microscopy (Konan non-contact, Robo, CA, USA) for endothelial cell counts, and slit lamp photography were obtained.

\section{Surgical technique}

Retrobulbar or peribulbar anaesthesia was used in all cases. A superior fornix-based conjunctival flap was made over approximately 2 clock hours. The first two cases were performed using a modified deep sclerectomy procedure in which a superficial scleral flap measuring $4 \times 4 \mathrm{~mm}$ was dissected into clear cornea. A second deeper flap measuring approximately $3 \times 3 \mathrm{~mm}$ was then dissected anteriorly until Schlemm's canal was encountered and unroofed. This deep flap was then excised just anterior to the canal. In the last four cases, a technique similar to that for trabeculotomy was adopted where, after forming the superficial scleral flap, a linear radial incision was made posteriorly from clear cornea beneath the scleral flap. This incision was slowly deepened until Schlemm's canal was identified.

Once Schlemm's canal was identified by either method, a small Vannas scissor was used to cut the opening of the canal externally to increase the size of the ostium. A viscocanalostomy canula ( $150 \mu \mathrm{m}$ outer diameter) was then inserted into the canal for a distance of approximately $5 \mathrm{~mm}$ to confirm a patent opening. The silicone tube ( $150 \mu \mathrm{m}$ outer diameter, 50 $\mu \mathrm{m}$ inner diameter) (Acritec, Germany) was bevelled and inserted approximately $5 \mathrm{~mm}$ into the cut end of Schlemm's canal. The other end of the tube was also bevelled anteriorly at a total tube length of about $8 \mathrm{~mm}$. A stab incision was then made through clear cornea, just anterior to trabecular meshwork with a sharp 27 gauge needle that approximated the outer tube diameter. The tube was then threaded through

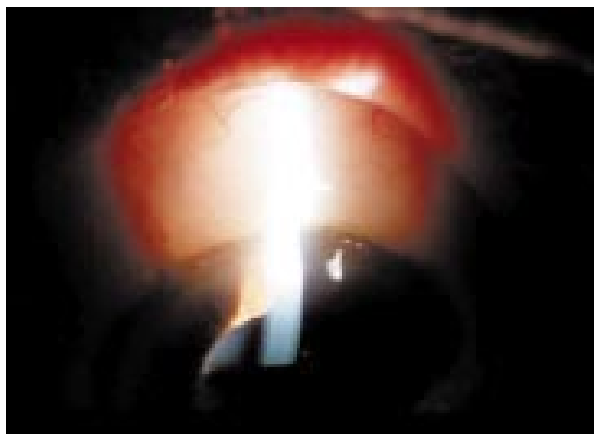

Figure 3 Slit lamp photography demonstrating a slight elevation of the conjunctiva consistent with a low diffuse filtering bleb. There is also evidence of conjunctival microcysts. The tube is not visible 19 months postoperative).

this opening into the anterior chamber (Fig 2). There was no attempt at tube occlusion or use of viscoelastic agents. The tube was secured to adjacent sclera with 10-0 Nylon suture in the initial cases, but not in the later ones. The superficial scleral flap was then sutured in place with 10-0 Nylon suture in as watertight a closure as possible. Conjunctiva was then sutured to the limbus. In the cases, in which flare measurements were performed, no subconjunctival injections were applied, in the other cases $2 \mathrm{mg}$ dexamethasone and $10 \mathrm{mg}$ gentamicin were subconjunctivally injected. Specific numeric target pressures were not set preoperatively in these patients. Postoperatively if the IOP range obtained was felt to be such that there was undue risk of glaucoma progression, medications were added.

\section{Main outcome measures}

Patients were evaluated at least on postoperative day 1 , weeks 1 and 2, and months 1, 2, 3, 6, and 9. Postoperative assessment included visual acuity, IOP measurement, slit lamp biomicroscopic examination and, in selected eyes, aqueous flare measurement, specular microscopy for endothelial cell counts, and slit lamp photography were obtained.

\section{RESULTS}

As an initial case series, of the six eyes in five patients included in this report, the tube was successfully implanted in five eyes of four patients. In one patient a 75 year old woman, the fifth of the series and the only patient with a diagnosis other than primary open angle glaucoma, who had an inflammatory glaucoma, Schlemm's canal could not be located at the time of surgery despite attempting both techniques of identification. The patient then underwent standard guarded filtration surgery. Additional data from this patient are not included.

Of the remaining four patients, all had primary open angle glaucoma, two were male, mean age was 66.5 years (range 38-80 years), three were white, one black. Surgeries were performed between July 2000 and April 2001. There were three right eyes and two left eyes with tube implantation. Follow up ranged from 3 days to 9 months. In one eye, the tube was explanted on the fourth postoperative day because of uncontrolled IOP presumed to be a result of intraoperative bleeding resulting in a hyphaema and tube obstruction with blood. Preoperatively the patient had been using aspirin that was not discontinued before surgery. In the four eyes with longer term follow up, the mean was 6.5 months with a range of 5-9 months.

Preoperative vision in the five implanted eyes ranged from 20/20 to hand movements. Preoperative mean IOP was 23.4 $\mathrm{mm} \mathrm{Hg}$ (range 19-35 $\mathrm{mm} \mathrm{Hg}$ ) with the use of an average of three glaucoma medications (range 2-4). Visual field loss varied from mild defect $(\mathrm{MD}-2.1 \mathrm{~dB}$ ) to severe with complete loss of central fixation. 
Table 1 Results of the five eyes with longer term experience with tube implantation with respect to intraocular pressure (IOP) and number of antiglaucoma medications used (meds).

\begin{tabular}{|c|c|c|c|c|c|c|c|c|c|c|}
\hline & \multicolumn{10}{|l|}{ Eye } \\
\hline & \multicolumn{2}{|l|}{1} & \multicolumn{2}{|l|}{2} & \multicolumn{2}{|l|}{3} & \multicolumn{2}{|l|}{4} & \multicolumn{2}{|l|}{5} \\
\hline & IOP & Meds & IOP & Meds & IOP & Meds & IOP & Meds & IOP & Meds \\
\hline Preop & 20 & 3 & 20 & 3 & 19 & 2 & 23 & 3 & 35 & 4 \\
\hline 1 day & 20 & 0 & 12 & 0 & 28 & 2 & 9 & 0 & 30 & 0 \\
\hline 3 days & 15 & 0 & 10 & 0 & 34 & 3 & 7 & 0 & 37 & 0 \\
\hline 1 week & 13 & 0 & 10 & 0 & & & 10 & 0 & 33 & 0 \\
\hline 2 weeks & 12 & 0 & 8 & 0 & & & 14 & 0 & 38 & 1 \\
\hline 4 weeks & 15 & 0 & 9 & 0 & & & 16 & 0 & 27 & 3 \\
\hline 6 weeks & 20 & 0 & & & & & 19 & 0 & 28 & 4 \\
\hline 3 months & 20 & 0 & 9 & 0 & & & 15 & 0 & 17 & 4 \\
\hline 6 months & 22 & 1 & 15 & 0 & & & 14 & 0 & 15 & 1 \\
\hline 9 months & 20 & 1 & & & & & 16 & 0 & & \\
\hline
\end{tabular}

Postoperative vision was little affected with no eye demonstrating any loss of best corrected vision of greater than two Snellen lines acuity at any time during follow up. Both hand movement eyes either remained unchanged or demonstrated slight improvement to counting fingers. Even on postoperative day 1 , two eyes were a single line worse, one a line better, and two unchanged.

Intraocular pressure was reduced from the preoperative level to a mean of $20 \mathrm{~mm} \mathrm{Hg}$ on postoperative day l (range 9-30 $\mathrm{mm} \mathrm{Hg}$ ) with only one eye having an increase in IOP. This was the case that required explantation on day 4 because of hyphaema. The remaining values for IOP and number of glaucoma medications are presented in Table 1. Of the four eyes with longer follow up, two demonstrated an immediate sustained substantial reduction of IOP with no need for glaucoma medications (eyes 2 and 3 ). In one eye (No 1 ) following an initial lowering of IOP for the first 4 weeks, IOP returned to near baseline, but without the need for any medication until 6 months postoperatively where a single medication was begun.

On biomicroscopic examination, of the four eyes with longer term follow up two exhibited low, diffuse filtering blebs (Fig 3). These two eyes were consistent with IOPs of $15 \mathrm{~mm}$ $\mathrm{Hg}$, despite absolutely tightness of the flap after surgery proved by irrigation of BSS though paracentesis. Additionally, in all four eyes there was evidence of conjunctival microcysts that were observed as soon as 1 week postoperatively. In a subset of two eyes, specular microscopy endothelial cell counts were obtained preoperatively and at approximately 5 months later. There was no evidence for any decrease in endothelial cell count at 5 and 9 months postoperatively (preop $=2500$, 2700 ; postop $=2550,2695)$. In these same eyes aqueous flare measurements revealed only a $15-25 \%$ increase in aqueous flare on postoperative day l compared to baseline, with this level returning to baseline by 1 week and remaining there through the postoperative course (data not shown).

\section{DISCUSSION}

This study reports the authors' initial experience with a novel approach to surgical glaucoma therapy. Specifically, this very small tube ( $150 \mu \mathrm{m}$ outer diameter) was chosen to allow a direct communication to be established between the anterior chamber and Schlemm's canal, effecting a trabecular bypass. There is evidence that supports the trabecular meshwork as the primary site for increased resistance to aqueous outflow in primary open angle glaucoma. ${ }^{10}{ }^{11}$ There is additional evidence that a small portion of the total outflow resistance is distal to the trabecular meshwork. ${ }^{12}$ This trabecular bypass tube can reduce resistance attributable to the meshwork, but not distal to it. Thus, the theoretical lower limit of IOP with the use of this tube is episcleral venous pressure. In many patients a reduction of IOP to this level, approximately 9-11 $\mathrm{mm} \mathrm{Hg}$ is sufficient. However, in cases where even lower pressures are desirable, an alternative approach would be necessary.

The canal is an oval that is reported to have dimensions of $30 \mu \mathrm{m} \times 280 \mu \mathrm{m}$. Intraoperatively, it was necessary to incise the wall of the canal to facilitate the placement of the tube ( $150 \mu \mathrm{m}$ outer diameter) within the lumen. The thin tube wall $(50 \mu \mathrm{m})$ also made this challenging. The length of tube within the canal was limited to $5 \mathrm{~mm}$ to prevent excessive resistance resulting from a longer tube length with the small lumen (50 $\mu \mathrm{m})$. The approach to identifying the canal as well as other portions of the technique varied as more experience was gained.

The standard filtering procedures-penetrating or nonpenetrating - are effective and well established in lowering the intraocular pressure in patients with glaucoma. However, the possible side effects like persistent hypotony, thin walled blebs with consecutive complications and as blebitis or endophthalmitis, and increasing IOPs over time are still challenging features. In addition, the need for lower IOPs than $15 \mathrm{~mm}$ $\mathrm{Hg}$ for patients with severe glaucoma forces us to use, up to now, some kind of antimetabolite, which causes the above mentioned complications. The ultimate goal of the new procedure would be to achieve IOPs around $12 \mathrm{~mm} \mathrm{Hg}$ without hypotony or/thin blebs. Since these first series of implants showed promising results, we are developing a slightly larger implant with a larger inner diameter.

This series represents the initial experience only and as more clinical understanding of the important factors in successful outcome are determined, the technique will continue to evolve.

\section{Authors' affiliations \\ D Spiegel, K Kobuch, Department of Ophthalmology, University of Regensburg, Regensburg, Germany}

Correspondence to: Professor Detlev Spiegel, Klinik und Poliklinik für Augenheilkunde der Universität Regensburg, Franz-Josef-Stras-Allee 11 , 93042 Regensburg, Germany; detlev.spiegel@klinik.uni-regensburg.de

Proprietary or commercial interests: None

Accepted for publication 10 June 2002

\section{REFERENCES}

1 Cairns JE. Trabeculectomy. Preliminary report of a new method. Am J Ophthalmol 1968;66:673-679.

2 Schwartz AL, Anderson DR. Trabecular surgery. Arch Ophthalmol 1974;92: 134-8. 
3 Stegmann R, Pienaar A, Miller D. Viscocanalostomy for open-angle glaucoma in black African patients. J Cataract Refract Surg 1999;25:316-22.

4 Mermoud A, Schnyder CC, Sickenberg M, et al. Comparison of deep sclerectomy with collagen implant and trabeculectomy in open-angle glaucoma. J Cataract Refract Surg 1999:25:323-31.

5 El Sayyad F, Helal M, El-Kholify $\mathrm{H}$, et al. Nonpenetrating deep sclerectomy versus trabeculectomy in bilateral primary open-angle glaucoma. Ophthalmology 2000;107:1671-4

6 Sunaric-Megevand G, Leuenberger PM. Results of viscocanalostomy for primary open-angle glaucoma. Am J Ophthalmol 2001;132:221-8.

7 Carassa RG, Bettin P, Fiori $M$, et al. Viscocanalostomy: a pilot study. Eur J Ophthalmol 1998;8:57-61.

8 Drusedau MU, von Wolff K, Bull H, et al. Viscocanalostomy for primary open-angle glaucoma: the Gross Pankow experience. J Cataract Refract Surg 2000;26:1367-73.
9 Jonescu-Cuypers C, Jacobi P, Konen W, et al. Primary viscocanalostomy versus trabeculectomy in white patients with open-angle . Ophthalmology

2001; 108:254-8.

10 Grant WM. Experimental aqueous perfusion in enucleated human eyes. Arch Ophthalmol 1963;69:738-801.

11 Johnson DH, Johnson M. How does nonpenetrating glaucoma surgery work? Aqueous outflow resistence and glaucoma surgery. J Glaucoma 2001;10:55-67.

12 Schuman JS, Chang W, Wang N, et al. Excimer laser effects on oufflow facility and oufflow pathway morphology. Invest Ophthalmol Vis Sci 1999:40:1676-80.

13 Spiegel D, Kobuch K, Hill RA, et al. Schlemm's canal implant: a new method to lower intraocular pressure in patients with POAG. Ophthalmic Lasers Surg 1999:30:492-4.

14 Spiegel D, Schefthaler, Kobuch K. Oufflow facilities through Descemet's membrane in rabbits [abstract]. Invest Ophthalmol Vis Sci 2000:41:S578.

\section{$\mathrm{ECHO}$}

\section{Major study points to better management for optic glioma}

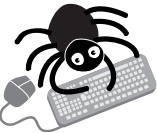

Please visit the British Journal of

Ophthalmology website [www. bjophthalmol. com] for link to this full article.

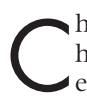

hildren with optic gliomas should benefit from better management after a study has established how different gliomas behave. Aggressive treatment is recommended for sporadic gliomas and

Seventeen cases in the study were of optic glioma associated with NFl and 17 were of sporadic optic glioma. Thirty one cases had an array of symptoms. Significantly more cases of sporadic glioma had visual symptoms (94\% versus $47 \%$ ) and died from their glioma (six versus two cases).

Diagnosis was generally based on histological findings whereas some cases with NFl were identified radiologically. Surgery was used twice as often as radiological treatment for both gliomas. Absolute mortality and five and 10 year survival were equivalent for both types, but a primary brain tumour developed in five cases of NFl, in three of which treatment for the glioma had been radiological.

The study was a retrospective case note study of optic glioma identified from the North West Regional NF1 Database and Manchester Children's Tumour Registry from records spanning more than 41 years. It was based on 34 cases with sufficient details of the natural history of the condition.

NFl is a common genetic disorder affecting 1 in 3000, and optic glioma is its most common complication, with a prevalence of $11-19 \%$. There are conflicting accounts of the natural history of optic gliomas and suggestions that gliomas in NFl are benign and screening is unnecessary, even that NFl protects children with optic glioma.

A Archives of Disease in Childhood 2002;87:65-70. 